\title{
The Intersection Between Geriatrics and Palliative Care: A Call for a New Research Agenda
}

\author{
Nathan E. Goldstein, MD and R. Sean Morrison, MD
}

Palliative care is interdisciplinary treatment focused on the relief of suffering and achieving the best possible quality of life for patients and their caregivers. It differs for geriatric patients from what is usually appropriate in a younger population because of the nature and duration of chronic illness during old age. In spite of the fact that death occurs far more commonly in older people than in any age group, the evidence base for palliative care in older adults is sparse. Over the coming years, the research foci in the field of geriatrics and palliative care that must be addressed include establishing the prevalence of symptoms in patients with chronic disease; evaluating the association between treatment of symptoms and outcomes; increasing the evidence base for treatment of symptoms; understanding psychological well-being, spiritual well-being, and quality of life of patients and elucidating and alleviating sources of caregiver burden; reevaluating service delivery; adapting research methodologies specifically for geriatric palliative care; and increasing the number of geriatricians trained as investigators in palliative care research. This article discusses specific methods to improve the current situation within each of these seven areas. J Am Geriatr Soc 53:1593-1598, 2005.

Key words: geriatrics; palliative care; research

M ultiple studies ${ }^{1-5}$ have reported a high prevalence of physical and psychosocial suffering, unmet practical and personal care needs, and high caregiver burden for persons living with serious, chronic, and terminal illness across all healthcare settings. The field of palliative care was developed to address the needs of patients and families that traditional medical care has not well met. Palliative care is interdisciplinary care focused on the relief of suffering and

From the Brookdale Department of Geriatrics and Adult Development, The Mount Sinai Medical Center, New York, New York; and The Geriatric Research, Education, and Clinical Center, Bronx Veterans Affairs Medical Center, Bronx, New York.

Address correspondence to Nathan E. Goldstein, MD, Brookdale Department of Geriatrics and Adult Development, The Mount Sinai Medical Center, Box 1070, One Gustave L. Levy Place, New York, NY 10029.

E-mail: Nathan.Goldstein@mssm.edu

DOI: $10.1111 / j .1532-5415.2005 .53454 . x$ achieving the best possible quality of life (QoL) - including ameliorating symptoms, relieving psychological distress, and promoting spiritual well-being - for patients with serious illness and their family caregivers. ${ }^{6}$ It is offered simultaneously with life-prolonging and curative therapies for persons living with serious, complex, and advanced illness (Figure 1). Several national organizations, including the Institute of Medicine (IOM) and the National Institutes of Health (NIH), have recently summarized the state of the science of palliative care and have called for increased research to help address the multiple unmet needs of patients and their families. This paper argues that the current evidence base and research priorities, as summarized in the $\mathrm{NIH}$ and IOM reports, ${ }^{4,5,7}$ are insufficient to meet the needs of older adults with serious, advanced, or terminal illness and in response, an agenda is outlined specifically for palliative care and aging research.

\section{The Need for Geriatric Palliative Care}

Despite the fact that death typically occurs in older adults in the setting of chronic illness, research in and clinical guidelines for palliative care have predominantly focused on younger adults - specifically those with cancer. The prototypical example of a palliative care patient is that of a 50year-old mother of two with advanced ovarian cancer. Care for this patient would include chemotherapy until it no longer meets the patient's goals of care, treating her symptoms (e.g., nausea, pain, fatigue), addressing her psychological and spiritual concerns, supporting her partner, and helping to arrange for care of her children after her death. The majority of this patient's care occurs at home (with or without hospice) or in the hospital, and the period of functional debility is brief (months). In reality, a frail 88 -year-old widowed woman with advanced heart failure, diabetes mellitus, osteoarthritis, mild cognitive impairment, and frailty typifies the most common example of a patient requiring palliative care. Palliative care for this patient involves treating the primary disease process (advanced heart failure), managing her multiple chronic medical conditions and comorbidities (diabetes mellitus, arthritis) and geriatric syndromes (cognitive impairment, frailty), assessing and treating the physical and psychological symptom distress associated with all of these medical issues, and establishing goals of care and treatment plans in the setting of an unpredictable prognosis. Additionally, the needs of her caregiver(s) are also dif- 


\begin{tabular}{|l|l|}
\hline \multicolumn{1}{|c|}{ Palliative Care } & \multicolumn{1}{c|}{ Geriatrics } \\
\hline Pain and symptom management & Frailty as indication for palliative care \\
\hline Communication skills and decision support & Functional assessment and effective interventions \\
\hline Bereavement and its importance & $\begin{array}{l}\text { Geriatric syndromes as sources of suffering and } \\
\text { their remediation }\end{array}$ \\
\hline $\begin{array}{l}\text { Role of palliative care in improving quality } \\
\text { of life and functional outcomes }\end{array}$ & Nursing home medicine \\
\hline Appropriate use of hospice & Sources of suffering in dementia \\
\hline
\end{tabular}

Figure 1. Overlapping topics shared by geriatrics and palliative care. Each topic, while originating in palliative care or geriatrics, crosses into the other field. For example, palliative care physicians often consider functional status, a concept fundamental to geriatrics, in helping patients make decisions about treatments. Likewise, training in geriatrics often includes education in pain and symptom management, concepts that are traditionally considered to be linked to palliative care.

ferent from those of the caregiver of the younger patient. Individuals caring for geriatric patients are often adult children with their own family, work responsibilities, and medical conditions, and these roles must be balanced with the months to years of personal care that they must provide to their aging parent. Finally, because older adults often make multiple transitions across care settings (home, hospital, rehabilitation, long-term care), ${ }^{8}$ especially in the last months of life, palliative care programs for older adults must assure that care plans and patient goals are maintained from one setting to another. (The topics shared by geriatrics and palliative care are outlined in Figure 2.)

\section{The Research Agenda for Palliative Care in Geriatrics}

Although death occurs far more commonly in older people than in any age group, the evidence base for palliative care in older adults is sparse. ${ }^{9}$ The areas of research that need to be addressed include establishing the prevalence of symptoms in patients with chronic disease, evaluating the association between symptom treatment and outcomes, increasing the evidence base for symptom treatment, understanding patients' psychological/spiritual well-being and QoL and elucidating sources of caregiver burden, reevaluating service delivery, adapting research methodologies specifically for palliative care, and increasing the number of geriatricians trained in palliative care research (Table 1).

\section{Establishing the Prevalence of Symptoms in Patients with Chronic Disease}

Although pain and symptom management is the cornerstone of modern palliative care, the majority of symptom

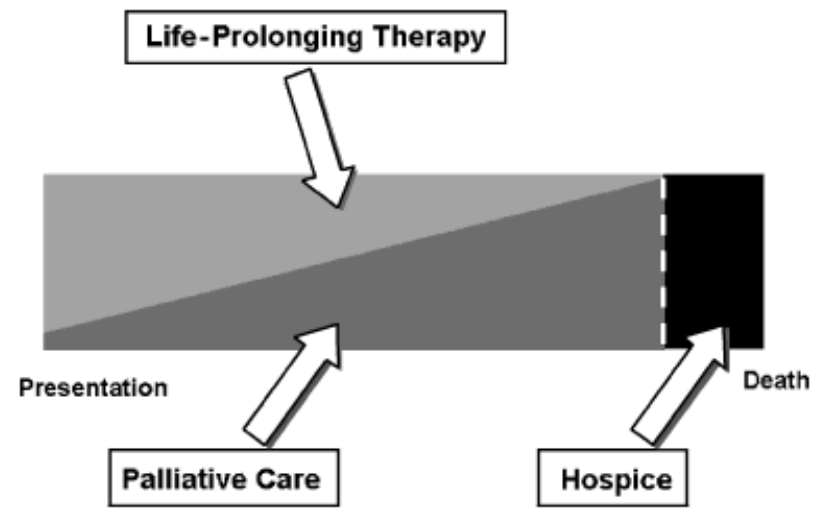

Figure 2. Palliative care is offered simultaneously with life-prolonging and curative therapies for persons living with serious, complex, and advanced illness. prevalence studies have focused on younger patients and have excluded patients with associated comorbidities and with cognitive impairment. Despite an increasing focus on pain research over the past decade, the precise incidence, prevalence, and etiologies of pain in older people is still not well described. Studies have suggested that the prevalence of significant pain in community-dwelling older adults may be as high as $25 \%$ to $56 \% .{ }^{10}$ Similarly, it has been suggested that $45 \%$ to $80 \%$ of nursing home residents have substantial pain and that many of these patients have multiple pain complaints and multiple potential sources of pain. ${ }^{11}$ Nevertheless, the etiology of these pain complaints, the type of pain (neuropathic vs somatic), and the association between pain and common geriatric disorders and syndromes (e.g., congestive heart failure, chronic obstructive pulmonary disease, frailty) has not been well established.

Available data from two large national studies in the United States and the United Kingdom point to a high prevalence of nonpain symptoms in adults with serious and chronic illness. ${ }^{1,12}$ In retrospective interviews with family members of patients who died of noncancer illnesses in the United Kingdom, investigators found that, whereas $67 \%$ of patients experienced moderate to severe pain, many had dyspnea $(49 \%)$, nausea $(27 \%)$, depression $(36 \%)$, and sleep disturbances (36\%). ${ }^{12}$ Similarly, the Study to Understand Prognoses and Preferences for Outcomes and Risks of Treatment, a study of 9,105 seriously ill hospitalized adults, found that more than $20 \%$ of patients with congestive heart failure, end-stage liver disease, lung cancer, or chronic obstructive pulmonary disease consistently experienced severe dyspnea during the 6 months before death. ${ }^{1}$ Neither of these studies enrolled many patients older than 75 .

Diagnosis-specific (e.g., pain prevalence studies in patients with heart failure, chronic lung disease) and syndrome-specific (e.g., studies examining prevalence of pain in frailty or dementia) investigations are needed to understand the symptom burden of older adults. Such studies could be primary investigations, or data could be obtained from ongoing clinical trials. For example, large randomized trials of treatments for advanced heart failure or early Alzheimer's disease could include comprehensive symptom assessment protocols to improve understanding of the symptoms associated with these diseases.

Assessing the Effect of Symptom Treatment on Outcomes Studies suggest that pain and other symptoms are underassessed and undertreated and are associated with a number 
Table 1. Limitations of Current Knowledge in Geriatric Palliative Care Research Areas and Steps for Improvement

Research Focus

1. Establish prevalence of symptoms

2. Associate treatment of symptoms with outcomes

\section{Improve evidence base for treating} symptoms

4. Understand psychological/spiritual well-being and quality of life of patients; elucidate sources of caregiver burden

\section{Reevaluate service delivery}

\section{Adapt research methods specifically for geriatric palliative care}

7. Increase number of geriatricians trained as investigators in palliative care research
Current Knowledge Limitations

Most studies focus only on cancer or acquired immunodeficiency syndrome, only evaluate pain, and do not focus on the oldest old.

Studies examining outcomes of pain management have outcomes of limited scope.

Few studies examining other symptoms.

Few studies support current practice for treatment of pain and nonpain symptoms.

Instruments developed to measure psychological / spiritual / quality-of-life outcomes may not be valid for older patients with long-term illness. Most studies examine caregiver strain in relationship to cancer or Alzheimer's disease.

Studies examining novel care delivery systems do not include frail adults with multiple comorbidities.

Local healthcare institutions have often not adapted or supported successful projects.

Criterion standard of patient self-report not always available, and observational scales are too complex.

Prognosis models not accurate in older patients with multiple chronic medical conditions.

Lack of trained investigators in palliative care with formal training in concepts fundamental to geriatrics.
Suggested Steps for Improvement

Further work to elucidate symptom burden in older adults with chronic disease as "stand-alone" studies or incorporated into existing studies with non-palliative care outcomes.

Design clinical trials examining the treatment of symptoms and their effect on geriatric outcomes such as function, gait, and frailty.

Add more-comprehensive symptom assessment to studies already examining such outcomes.

Design clinical trials to determine best treatment modalities for symptom management.

Validate former instruments or develop new ones to assess spiritual and psychological assessment.

Design observational studies to further elucidate caregiver burden in patients with chronic illness followed by intervention studies to alleviate burden.

Expand study inclusion criteria to include broader range of patients more representative of geriatric population as a whole.

Create financial incentives to foster sustainable projects.

Create new scales or modify existing ones that can be validated in this population.

Develop models that can predict prognosis taking into account multiple comorbidities in aging individuals.

Encourage palliative care fellowships to include geriatric training and include a research-focused training mission. of negative outcomes. Untreated pain has been associated with depression, decreased socialization, impaired ambulation, and increased healthcare usage, ${ }^{9}$ but the results of the majority of these studies are difficult to extrapolate to older adults because they typically enroll younger subjects, and when they enroll older adults, these subjects are relatively healthy with defined uncomplicated conditions (e.g., osteoarthritis, postoperative pain). Studies have typically not included frail older adults with multiple comorbidities and have not examined the association between pain and its treatment and important geriatric outcomes such as function, falls, cognition, and frailty. ${ }^{9}$ Almost no studies have examined the association between nonpain symptoms and outcomes in older patients.

Designing clinical trials specifically focusing on symptoms and their effect on outcomes would be one way to bridge this knowledge gap. Another possibility would be to add more-comprehensive symptom assessment to studies already examining some of these outcomes. For example, a study designed to examine the role of physical therapy in falls prevention in adults aged 80 and older could also include a detailed symptom scale to determine how symptoms such as pain, fatigue, and breathlessness relate to the primary outcome measure of falls.

Improving the Evidence Base for Treatment of Symptoms The evidence base for the effective management of pain and other symptoms in older adults is sparse. Although the American Geriatrics Society recently published guidelines for the treatment of acute and persistent pain, these guidelines were largely based upon small cohort studies and expert opinion. ${ }^{13}$ Recommendations for age-adjusted dosing are not available for most analgesics, ${ }^{9}$ and almost all analgesics have side-effect profiles that are particularly prob- 
lematic or may be accentuated in older adults (e.g., constipation, delirium, renal failure). ${ }^{13}$ Studies of interventions directed at other symptoms have rarely included older adults or have focused on narrow conditions (e.g., chemotherapy-induced nausea), thus the results are difficult to generalize to older adults with advanced illness and multiple comorbidities. ${ }^{9}$

One reason that the evidence base for treating pain in older patients is weak is that there is a paucity of validated assessment tools. There are no well-studied instruments that can be used efficiently to evaluate pain in the older population, especially given the fact that patients may have multiple types, locations, and etiologies of pain. The older woman mentioned earlier might have pleuritic chest pain from pneumonia, anginal pain from coronary ischemia, diabetic neuropathy in her feet, and diffuse body aches secondary to heart failure. The standard symptom assessment scale asking this patient to "rate her pain on a scale of 1 to 10 " would miss the nuances that would be necessary to treat her effectively. This assessment would be made even more difficult were she to have severe cognitive impairment, a subset of patients for which good assessment tools do not exist.

\section{Understanding Patients' Psychological/Spiritual Well- Being and QoL: Elucidating Sources of Caregiver Burden} Although research in palliative care has been done to understand factors important to dying patients' psychological and spiritual well-being, little of this work has focused on geriatric patients. Because older adults have multiple chronic diseases, the ways they cope with illness may be different from how younger individuals with a sudden life-threatening illness cope. Although there are QoL measurements that have been used in palliative care, these tools are often too burdensome to use for older patients, because they have typically been developed in younger patient populations who usually do not have multiple comorbidities. ${ }^{14}$ Similar arguments can be made in terms of work done to understand the psychological well-being of patients. ${ }^{15,16}$ Spiritual assessment of patients has been most often explored in patients with cancer who are near the end of life. ${ }^{17} \mathrm{It}$ is unclear whether older patients who have been coping with chronic illness for an extended period of time have the same spiritual needs as the younger patients whose death is imminent who are typically included in studies examining religiosity.

Palliative care for older patients involves treating the family as well as the patient, and as such, there needs to be an increased focus on the needs of caregivers and methods to alleviate the burden that this role places upon them. Studies have shown that caregivers who care for patients near the end of life have high levels of anxiety and depression and higher rates of mortality. ${ }^{2,3}$ The majority of work examining caregiver burden has been conducted in patients with dementia or cancer, and the caregiver is often the spouse. Research still needs to be conducted to examine burden in caregivers of geriatric patients with end-stage organ failure, especially given that these individuals tend to be adult children with families of their own. Similarly, the majority of scales used to assess caregiver burden have been developed for patients with dementia ${ }^{18}$ or cancer; ${ }^{19}$ older patients with heart or lung disease have different care needs.
Methods of alleviating caregiver burden are not well studied, and interventions to reduce caregiver stress need to be developed.

\section{Reevaluating Service Delivery}

The current patterns of care delivery and reimbursement fail to address many of the needs of older patients with serious chronic illness. ${ }^{20}$ Medicare covers acute hospitalizations, physician services, rehabilitation, and short-term posthospital care, and as such it is ill-equipped to respond to the long-term needs of the chronically ill. Since 1983, the Medicare hospice benefit also provides care to patients, but because of various restrictions, only a minority of people with life-threatening illness can benefit from this system. ${ }^{21}$ The vast majority of patients with serious and chronic illness have uncertain prognoses and poorly predictable disease trajectories and require care beyond that typically provided by acute care hospitals, physician office visits, and hospice. ${ }^{22}$

Several forms of care delivery have been shown to decrease costs and improve outcomes, but much of the data supporting these projects are incomplete from the point of view of older patients. For example, inpatient hospital units dedicated to frail older adults have been shown to improve satisfaction and reduce functional decline, discharge to nursing home, and the incidence and duration of delirium, ${ }^{23-25}$ but these studies have typically not included frail older adults with multiple comorbidities, ${ }^{23,24}$ have not focused on interventions directed at pain and other symptoms (except for those examining delirium), ${ }^{25}$ and have rarely included measures of health-related QoL outside of functional status. ${ }^{24,25}$ Although nurse-based disease-management programs that coordinate home care services for patients with chronic conditions (e.g., heart failure, acquired immunodeficiency syndrome) have been associated with significant reductions in mortality, hospital readmissions, physician office visits, emergency visits, and admissions to nursing homes and greater satisfaction, ${ }^{26,27}$ these studies have been disease specific, have not included symptom interventions, and have not focused specifically on measures related to QoL.

To change reimbursement patterns, novel demonstration programs must be developed to fund care for patients with chronic illness. Some programs, such as those within the Veterans Affairs healthcare system and the Program of All-Inclusive Care for the Elderly have been shown to be effective in terms of improving care for older adults, ${ }^{21}$ but these programs are not easily adapted to other settings. In addition, once successful demonstration programs are completed, a local healthcare institution must adopt them so that they may become sustainable.

\section{Adapting Research Methodologies Specifically for Geriatric Palliative Care}

Successful research in palliative care for older adults will require the development of new research instruments, designs, and analytic techniques. Traditionally, research in pain and other symptoms has relied upon patient self-report as the criterion standard of assessment. ${ }^{28}$ Self-report is particularly problematic in geriatric patients with cognitive impairment, and reliable means of assessing pain and other 
symptoms through behavioral observation or through the use of proxies are required. Unfortunately, existing observational scales require considerable skill to administer, and validated scales employing proxy respondents are not available. ${ }^{9}$ A fundamental difference between "traditional" palliative care and palliative care for older patients relates to the multiple treatments for various conditions. This causes extra complexities for researchers studying older patients because it is often difficult to determine the etiology of some symptoms and to distinguish symptoms caused by the patient's illness from those resulting from treatments. Although a few such instruments to do this exist, they are relatively complex and burdensome and have not been validated in older adults. ${ }^{29,30}$ Finally, although some research questions in geriatric palliative care may be addressed using the criterion standard of clinical research - the randomized controlled trial-many others may only be addressed through observational or nonrandomized studies. ${ }^{4}$

The issue of prognosis is of particular importance in geriatric palliative care, and there needs to be an increased focus of research on improving methods to predict prognosis in patients with chronic disease. Even though predictive models can result in an accurate survival curve for a large group of people with various conditions such as organ failure and cancer, these models cannot accurately predict prognosis for individual patients. ${ }^{31}$ This becomes even more complicated in geriatric patients, who may have multiple illnesses, making it even more difficult to predict the ultimate cause and timing of death. In addition, miscommunication about prognosis in older patients often occurs. ${ }^{32}$ Understanding prognosis is particularly important in older patients because it can be a key piece of information affecting how these individuals make decisions. Although geriatricians are trained in balancing benefits and burdens of interventions for older patients, research needs to be done to elucidate the ways that patients and clinicians work together to make decisions about treatments.

Specific analytical problems unique to palliative care in older adults also require attention. For example, the problem of missing or distorted data is considerable in palliative care research; ${ }^{4}$ this may be particularly true for older adults with advanced illness, who may be enrolled in research trials but may die without being questioned. Patients with baseline cognitive impairment, even if subtle, may be more vulnerable to being confused or unconscious after receiving treatments and thus may not be able to be questioned directly about their symptoms or attitudes. In general, there is little research to guide choices in imputing or substituting data, and this is even more important for older palliative care patients because of the greater amount of data missing from these patients. Similarly, nonmortal outcomes are often of considerable importance in palliative care but may be difficult to analyze given the high death rates in the population being studied. Thus, techniques that employ or evaluate competing risks in the context of survival analyses may need to be employed or further developed. ${ }^{33}$ Finally, because many studies in palliative care may be observational or employ quasi-experimental designs, expertise in specific analytical techniques that are directly applicable to these types of studies (e.g., hierarchical linear modeling) is often required. ${ }^{33}$

\section{Training Geriatricians to Become Investigators in Palliative Care Research}

There is a pressing need to train geriatricians in palliative care research. The IOM report on improving palliative care for cancer patients cited the lack of trained investigators and established research groups as a major impediment to the conduct of quality palliative care research. ${ }^{5}$ As of 2004, there were approximately 50 funded fellowship training programs available in the United States, of highly variable quality, size, and outcomes. ${ }^{34}$ Most are small ( 1 or 2 fellows), 1-year clinical programs and follow a nonacademic model of education and training. ${ }^{35}$ Conversely, although strong research training programs in geriatrics are available, there are few established senior researchers in palliative care that can mentor and support junior investigators adequately. Palliative care research tends to focus on a specific type of care at a specific point in time (e.g., care for cancer patients near the end of life), but with the changing demographics in society, there will be a need for researchers well versed in the fields of geriatrics and palliative care who can think of the importance of competing comorbidities and how these will affect a patient's care over time.

\section{CONCLUSION}

Substantial data suggest that the care of older adults living with serious illness is in need of improvement. Although great strides were accomplished in outlining the need for improving palliative care at the recent NIH State of the Science Conference on Improving End-of-Life Care, ${ }^{7}$ even at this landmark event there was a focus on terminal disease and not on expanding the idea of palliative care to include improving the quality of care of older patients with multiple chronic diseases. If the field of geriatrics is to encompass the needs of all older adults, it needs to actively integrate within it the principles of palliative care and promote and support the development of new investigators in palliative care research. By 2030, it is predicted that $20 \%$ of the U.S. population will be aged 65 and older, ${ }^{4}$ and by 2050 , it is estimated that there will be 80 million Americans aged 65 and older. The increase in numbers of older Americans is on the horizon; now is the time to improve research in geriatric palliative care to be able to meet their needs.

\section{ACKNOWLEDGMENTS}

Financial Disclosures: Dr. Goldstein is a Hartford Center of Excellence Scholar. Dr. Morrison is the recipient of a MidCareer Investigator Award in Patient-Oriented Research (K24AG022345) from the National Institute on Aging.

Author Contributions: Both authors had an equal role in the design, preparation, and final approval of this manuscript.

Sponsor's Role: None.

\section{REFERENCES}

1. Phillips RS, Hamel MB, Covinsky KE et al. Findings from SUPPORT and HELP. An introduction. Study to Understand Prognoses and Preferences for Outcomes and Risks of Treatment. Hospitalized Elderly Longitudinal Project. J Am Geriatr Soc 2000;48(5 Suppl):S1-S5.

2. Schulz R, Newsom J, Mittelmark M et al. Health effects of caregiving: The caregiver health effects study: An ancillary study of the Cardiovascular Health Study. Ann Behav Med 1997;19:110-116. 
3. Schulz R, Beach S. Caregiving as a risk factor for mortality. The Caregiver Health Effects Study. JAMA 1999;282:2215-2219.

4. Field MJ, Cassel CK, eds. Approaching Death. Improving Care at the End of Life. Washington, DC: National Academy Press, 1997.

5. Cleeland CS. Cross-cutting research issues. A research agenda for reducing distress of patients with cancer. In: Foley K, Gelband H, eds. Improving Palliative Care for Cancer. Washington, DC: National Institute of Medicine, 2001, pp 233-274.

6. Clinical Practice Guidelines for Quality Palliative Care. Brooklyn, NY: National Consensus Project for Quality Palliative Care, 2004.

7. NIH. State-of-the-Science Conference on Improving End-of-Life Care [online]. Available at http://consensus.nih.gov/ta/024/024EndOfLifepostconfINTRO.htm Accessed March 9, 2005.

8. Coleman EA, Boult C. Improving the quality of transitional care for persons with complex care needs. Position statement of the American Geriatrics Society health care systems committee. J Am Geriatr Soc 2003;51:556-557.

9. Morrison RS, Meier DE, eds. Geriatric Palliative Care. New York: Oxford University Press, 2003.

10. Helm RD, Gibson SJ. Pain in Older People. In: Cronbie IK, Croft R, Linton SJ et al., eds. Epidemiology of Pain. Seattle, WA: IASP Press, 2000, pp 102-112.

11. Ferrell B. Pain evaluation and management in the nursing home. Ann Intern Med 1995;123:681-687.

12. Seale C, Cartwright A. The Year Before Death. Brookfield, VT: Ashgate Publishing Co., 1994.

13. American Geriatrics Society. The management of persistent pain in older adults [on-line]. Available at www.americangeriatrics.org Accessed January 25, 2004.

14. Schwartz CE, Merriman MP, Reed G et al. Evaluation of the Missoula-VITAS quality of life index-revised: Research tool or clinical tool? J Palliat Med 2005;8:121-135.

15. Lyness JM. End-of-life care: Issues relevant to the geriatric psychiatrist. Am J Geriatr Psychiatry 2004;12:457-472.

16. Emanuel EJ, Fairclough DL, Wolfe P et al. Talking with terminally ill patients and their caregivers about death, dying, and bereavement: Is it stressful? Is it helpful? Arch Intern Med 2004;164:1999-2004.

17. Murray SA, Kendall M, Boyd K et al. Exploring the spiritual needs of people dying of lung cancer or heart failure: A prospective qualitative interview study of patients and their carers. Palliat Med 2004;18:39-45.

18. Zarit SH, Reever KE, Bach-Peterson J. Relatives of the impaired elderly: Correlates of feelings of burden. Gerontologist 1980;20:649-655.

19. Weitzner MA, Jacobsen PB, Wagner H Jr et al. The Caregiver Quality of Life Index-Cancer (CQoLC) scale: Development and validation of an instrument to measure quality of life of the family caregiver of patients with cancer. Qual Life Res 1999;8:55-63.
20. Lynn J, Schuster JL, Kabcenell A. Improving Care for the End of Life. A Sourcebook for Health Care Managers and Clinicians. New York: Oxford University Press, 2000.

21. Morrison RS, Meier DE. Clinical practice. Palliative care. N Engl J Med 2004;350:2582-2590.

22. Knaus WA, Harrell FE Jr, Lynn J et al. The SUPPORT prognostic model. Objective estimates of survival for seriously ill hospitalized adults. Study to Understand Prognoses and Preferences for Outcomes and Risks of Treatments. Ann Intern Med 1995;122:191-203.

23. Cohen HJ, Feussner JR, Weinberger M et al. A controlled trial of inpatient and outpatient geriatric evaluation and management. N Engl J Med 2002; 346:905-912.

24. Landefeld CS, Palmer RM, Kresevic DM et al. A randomized trial of care in a hospital medical unit especially designed to improve the functional outcomes of acutely ill older patients. N Engl J Med 1995;332:1338-1344.

25. Inouye SK, Bogardus ST Jr, Charpentier PA et al. A multicomponent intervention to prevent delirium in hospitalized older patients. $\mathrm{N}$ Engl J Med 1999;340:669-676.

26. Riegel B, Carlson B, Kopp Z et al. Effect of a standardized nurse case-management telephone intervention on resource use in patients with chronic heart failure. Arch Intern Med 2002;162:705-712.

27. Elkan R, Kendrick D, Dewey M et al. Effectiveness of home based support for older people: Systematic review and meta-analysis. BMJ 2001;323: 719-725.

28. Agency for Health Care Policy and Research Management of cancer pain: Adults. Cancer Pain Guideline Panel. Am Fam Physician 1994;49: 1853-1868.

29. Portenoy RK, Thaler HT, Kornblith AB et al. The Memorial Symptom Assessment Scale. An instrument for the evaluation of symptom prevalence, characteristics and distress. Eur J Cancer 1994;30A:1326-1336.

30. Dudgeon D, Raubertas RF, Rosenthal SN. The short-form McGill Pain Questionnaire in chronic cancer pain. J Pain Symptom Manage 1993;8:191-195.

31. Levenson JW, McCarthy EP, Lynn J et al. The last six months of life for patients with congestive heart failure. J Am Geriatr Soc 2000;48(5 Suppl):S101-S109.

32. Fried TR, Bradley EH, O’Leary J. Prognosis communication in serious illness. Perceptions of older patients, caregivers, and clinicians. J Am Geriatr Soc 2003;51:1398-1403.

33. Penrod JD, Morrison RS. Challenges for palliative care research. J Palliat Med 2004;7:398-402.

34. American Academy of Hospice and Palliative Medicine. Fellowship Program Directory. AAHPM [on-line]. Available at www.aahpm.org/fellowship/directory/htm Accessed November 30, 2004.

35. Casarett DJ. The future of the palliative medicine fellowship. J Palliat Med 2000;3:151-155. 